\title{
EMG-based learning approach for estimating wrist motion
}

\author{
S. El-Khoury, ${ }^{1}$ I. Batzianoulis, ${ }^{1}$ C. W. Antuvan, ${ }^{2}$ S. Contu, ${ }^{2}$ L. Masia, ${ }^{2}$ S. Micera ${ }^{3}$ and A. Billard ${ }^{1}$
}

\begin{abstract}
This paper proposes an EMG based learning approach for estimating the displacement along the 2-axes (abduction/adduction and flexion/extension) of the human wrist in real-time. The algorithm extracts features from the EMG electrodes on the upper and forearm and uses Support Vector Regression to estimate the intended displacement of the wrist. Using data recorded with the arm outstretched in various locations in space, we train the algorithm so as to allow robust prediction even when the subject moves his/her arm across several positions in space. The proposed approach was tested on five healthy subjects and showed that a $R^{2}$ index of $63.6 \%$ is obtained for generalization across different arm positions and wrist joint angles.
\end{abstract}

\section{INTRODUCTION}

Electromyography (EMG) signals provide information related to the muscles' activities and have been used for the user's motion intention estimation. In particular, several EMG based systems were proposed for estimating the hand and wrist motions and were used consequently as an interface for controlling wrist exoskeletons [1], [2], or prosthetic devices used in the hand and wrist [3], [4], for teleoperating robotic arms [3], [5], or in a virtual environment to control computer-animated hands [6].

In order to estimate and control the wrist motion, people have primarily focused on classification-based methods. For instance, in [7], the authors compare several classification methods for discriminating between six gestures (hand open, hand close, wrist flexion, wrist extension, supination and pronation gestures). In [3], [5], the authors use different machine learning techniques to discriminate the EMG patterns related to eight different gestures, adding to the previous six gestures the wrist abduction and adduction movements. Similarly, in [4], [6], [8], the authors propose methods for classifying ten different types of movements including flexion/extension of the wrist as well as flexion/extension of a different combination of fingers. All these approaches investigate a discrete classification of the wrist or hand/fingers opening and closing.

In [9], the author addresses the question of prosthesis design and acceptance from the consumers' perspective and points out their need to control the wrist. Once the grasp is

\footnotetext{
*This work has received funding from the Swiss National Science Foundation through the National Centre of Competence in Research (NCCR) in Robotics

${ }^{1} \mathrm{~S}$. El-Khoury, I. Batzianoulis and A. Billard are with Learning Algorithms and Systems Laboratory (LASA)at Ecole Polytechnique Federale de Lausanne (EPFL), Switzerland

${ }^{2}$ C. W. Antuvan, S. Contu and L. Masia are with Assistive Robotics and Interactive Ergonomic Systems Laboratory (ARIES) at Nanyang Technological University, Singapore

${ }^{3} \mathrm{~S}$. Micera is with Translational Neural Engineering Laboratory (TNE) at Ecole Polytechnique Federale de Lausanne (EPFL), Switzerland and with the BioRobotics Institute at Scuola Superiore SantAnna, Pisa, Italy
}

performed, and in order to manipulate the corresponding object without having to move the whole arm, one needs to be able to control the wrist joint angles smoothly and continuously which could not be ensured through classification techniques. Some researchers [10], [11] focused on continuously estimating the arm motion and used standard supervised machine learning algorithms to create a mapping between muscles' activities in the arm/forearm and either the forearm $6 D$ position/orientation [10] or the 4 rotational degrees of freedom modelling the joints of the shoulder and elbow [11]. However, these approaches did not consider the wrist joint angles. The authors in [1], [2] did take into account the motion of the wrist in order to estimate the user's intended torque to control a wrist exoskeleton. In their experiment, the users' forearm rested on one plate and their hands on a second plate. Two rigs were designed to record the level of torque for flexion/extension and for abduction/adduction deviations of the wrist. These recordings were divided into different sets corresponding each to a percentage of the maximum voluntary contraction (MVC) in each direction. No continuous motion was estimated but a classification technique was employed to distinguish between these different classes. In [12], the authors proposed an algorithm for a simultaneous estimation of the three degrees of freedom of the wrist. They also showed the applicability of their approach to unilateral amputees by employing a bilateral mirror-training strategy [13]. In order to estimate the wrist movements, the authors measured the muscles activities by placing 7 pairs of electrodes all around the thickest region of the forearm and the subjects were seated and resting their elbows on an armrest. This paper proposes a similar approach to [12] by estimating the wrist movements from the muscles activities of the upper limb. The main contribution of this work is related to two points; for a more intuitive control/estimation of the wrist movements, the subjects performed the experiment without restraining the motion of their elbows. Given this freedom of motion, we also report on generalization of wrist motion estimation from one position of the arm to another. The rest of this paper is organized as follows. Section 2 details our setup. Section 3 is dedicated to the wrist motion estimation and section 4 concludes.

\section{A. Participants}

\section{Methods}

Five healthy subjects, age between $23-28$, participated in the experiment. All volunteers have been informed about the experimental aim of the study and gave their consent. The experimental procedures involving human subjects described in this paper were approved by EPFL's Ethical Committee. All subjects performed the experiments with their right hand 


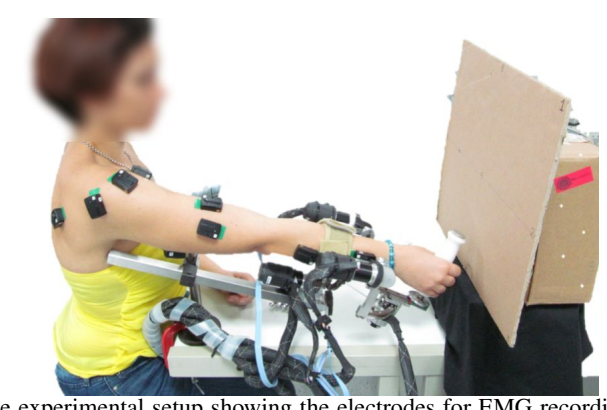

Fig. 1: The experimental setup showing the electrodes for EMG recording and the vision tracking system for computing the arm joint angles.

TABLE I: Muscles which activity is captured.

\begin{tabular}{c|c} 
& Muscles \\
\hline 1 & Deltoid Medial (DMED) \\
2 & Deltoid Anterior (DANT) \\
3 & Deltoid Posterior (DPOS) \\
4 & Biceps Brachii long heads (BICL) \\
5 & Triceps Brachii long head (TRIC) \\
6 & Infraspinatus (INFRA) \\
7 & Brachialis (BRA) \\
8 & Flexor Digitorum Superfialis (FLDS) \\
9 & Extensor Digitorum Communis (EXDC) \\
10 & Flexor Carpi Ulnaris (FLCU) \\
11 & Extensor Carpi Ulnaris (EXCU)
\end{tabular}

(dominant hand [14]), and they had no prior history of neurological disorders and neuromuscular injuries.

B. Apparatus

Figure 1 illustrates the experimental setup showing the electrodes for EMG recording and the WRIST device [15] system for capturing the wrist joint angles [16]. The robot allow full range of motion for the human wrist and measures wrist rotations on the three axes by means of digital encoders with a resolution of $4098 \mathrm{bit} / \mathrm{turn}$.

Raw surface EMG signals of the upper limb muscles, (Tables I), were collected using the Delsys Trigno Wireless EMG system, with sampling rate at $1000 \mathrm{~Hz}$. The choice of these muscles and the placement of the corresponding electrodes is based on previous studies related to the estimation of the upper limb motion [11]. The raw data were filtered with a seventh-order band-pass Butterworth filter with cutoff frequencies of $50 \mathrm{~Hz}$ and $500 \mathrm{~Hz}$ for suppression of movement artifact. To construct a linear envelope, fullwave rectification was performed, followed by smoothing with a low-pass seventh-order Butterworth filter with cutoff frequency of $20 \mathrm{~Hz}$. In addition, the resulting EMG signal was normalized with the maximum voluntary contraction (MVC).

\section{Experimental Protocol}

Experiments were performed by 5 subjects with their dominant arm. During the experiment, each subject, was instructed to extend and move their arm to one of 9 predefined directions (these directions correspond to different positions on a planar square $40 \mathrm{~cm} \times 40 \mathrm{~cm}$ grid) and then to explore their wrist configuration space by performing circular motions clockwise. These circular motions were chosen in order to make sure that the subjects were exploring the whole reachable space of their wrist by holding the rest of the arm fixed, and in this sense the experiment was similar to the one performed in [12] where the subjects also performed circular motions. The number of circular motions was set to 30 in order to avoid the subject's fatigue.
III. WRIST JOINT ANGLES ESTIMATION

We learn a mapping between the joint angles at the wrist and the EMG signals of the arm. After the data acquisition is performed, it is organised into a set of (samples, targets). Each sample consists of values of the EMG electrodes averaged over a moving window $w=200 \mathrm{~ms}$ with an overlap of $100 \mathrm{~ms}$ as well as the relative proportion of one EMG signal with respect to all other signals, and the targets are the 3 corresponding wrist joint angles. The resulting (samples, targets) pairs are used to train 3 Support Vector Regression models $(S V R)$ with Gaussian kernels, each corresponding to a wrist joint angle. SVR method was chosen due to its high performance in non-linear estimation and fast convexity for real time implementation. For training an $S V R$ model, the corresponding data is divided into a training data set $(80 \%$ of the samples) and a testing set (20\% of the samples). A grid search is performed to select the hyperparameters, $\varepsilon$, cost and kernel width along with a 10 -fold cross validation procedure for computing the corresponding regression errors on the training and test data sets. Three different mappings between the EMG signals and the wrist joint angles were performed; 1) mapping taking into consideration all EMG signals in the upper arm and forearm (electrodes 1 to 11 in table (IV)); 2) mapping from the EMG signals in the upper arm only (electrodes 1 to 7 in table (IV));3) mapping obtained using EMG signals in the forearm arm only (electrodes 8 to 11 in table (IV)). To evaluate the regression, the $R^{2}$ index was computed as follows [17]:

$$
R^{2}=\frac{\left(n \sum_{i=1}^{n} f\left(x_{i}\right) y_{i}-\sum_{i=1}^{n} f\left(x_{i}\right) \sum_{i=1}^{n} y_{i}\right)^{2}}{\left(n \sum_{i=1}^{n} f\left(x_{i}\right)^{2}-\left(\sum_{i=1}^{n} f\left(x_{i}\right)\right)^{2}\right)\left(n \sum_{i=1}^{n} y_{i}^{2}-\left(\sum_{i=1}^{n} y_{i}\right)^{2}\right)}
$$

Where $n$ is the number of (samples, targets) data points, $x_{i}$ is a sample, $y_{i}$ is its corresponding target, and $f\left(x_{i}\right)$ is the value predicted by the regression model.

Three regression tests were performed:

- The first one aims at testing whether good results could be obtained by training a $S V R$ model on a subset of the data $(80 \%)$ obtained from one arm position and then testing the model on the remaining $(20 \%)$. Corresponding results are shown in the first row of tables (II IV).

- The second one aims at testing the generalization of the trained algorithm across arm positions. In this case, the training was performed either on one, two, three, four and five arm positions, and the testing was conducted on data obtained from the remaining eight, seven, six, five and four arm positions. The training arm positions were selected randomly among the nine different arm positions available from the experimental setup. This procedure is repeated nine times and the results shown in rows 2 to 6 in tables $(\mathrm{II}[\mathrm{IV}$, correspond to the average and standard deviation of the $R^{2}$ index across these different nine trials.

- We also tested the ability of generalization of the regression model from one day to the other. One subject performed the same experiment described previously 
across three days. Tables (V VII summarize the results obtained by training the model on data collected from one or two days and testing it on the remaining days.

\section{Discussion}

In this section, we presented a method for estimating the wrist joint angles using EMG signals from the upper limb. Results were computed per subject and the learned regression models were not generalizable across subjects.

In a first step, we aimed at testing whether a good regression model estimating the wrist joint angles for a specific free arm position with no restrictions on the elbow motion could be obtained. Nine arm positions were tested for each subject and tables $(\mathrm{II}, \mathrm{III}, \mathrm{IV})$ summarize the corresponding results, showing that on average, the $R^{2}$ index is of $77.7 \%$ for the testing set across the wrist joints angles and across the 9 arm positions when muscles of the whole upper limb are taken into account, of $72.4 \%$ when only 4 muscles in the forearm are taken into account, and of $67.9 \%$ when only muscles in the upper arm were taken into consideration. These results are comparable to the ones obtained in [12] where the authors obtained an $R^{2}$ index of $72.0 \%$ for healthy subjects performing circular motions. In order to maintain a correct alignment of the wrist device axes across different positions of the arm, the forearm was fixed during the experiment which obstructed the motion of the pronation/supination of the wrist. Consequently, only two angles were predicted by the regression model, the abduction/adduction and flexion/extension angles. Since we do not take into consideration the pronation/supination angle which is the most difficult to predict because of the deep location of the corresponding muscles, our results are slightly higher than the ones in [12]. We also noticed that good results were obtained on the testing set from muscles activities in the upper arm. This is encouraging if the targeted application is to control a myoelectric wrist exoskeleton for unilateral transradial amuptees, as a mirrored bi-lateral contraction could be employed for training as detailed in [12].

In a second step, we trained a regression model on a number of arm positions and tested the model on the remaining positions. Good generalization results were obtained and summarized in tables (II] III] IV). On average, the generalization $R^{2}$ index is of $63.6 \%$ across the wrist joints angles when the model is trained on 5 arm positions and when muscles of the whole upper limb are taken into account and of $58.1 \%$ and of $49.2 \%$ when respectively only 4 muscles in the forearm and only muscles in the upper arm are taken into account. We notice that better generalization results are obtained when the model is trained on an increasing number of arm positions.

Finally, we tested the ability of the model to generalize across several days while performing the experiment on the same subject. Results are summarized in tables (V) VII ). These results show that the data obtained from days 1 and 3 are similar and thus a good generalization is obtained by training the model on the data collected from one day and testing it on the other. Table (VII) shows that a $R^{2}$ index of $52.9 \%$ is obtained across wrist angles when all muscles are taken into account and when training the model on the data collected from day 1 and testing it on the data collected from day 3. Similarly, table (V) shows that a $R^{2}$ index of $48.1 \%$ is obtained when the training is performed on the data collected from day 3 and tested on the data collected from day 1 . The data collected from day 2 was not correctly predicted from the model trained on the other two days when taking into account all muscles, see table (VI). Better results were obtained when taking into account muscles from the upper arm. Although these results seem encouraging, the ability of the model to generalize should be tested in more subjects. Further research could focus to real time implementation for investigating the efficiency of the regression performance to both healthy subjects and amputees.

\section{REFERENCES}

[1] Z. O. Khokhar, Z. G. Xiao, and C. Menon, Surface EMG pattern recognition for real-time control of a wrist exoskeleton, in BioMedical Engineering OnLine, vol. 9, pp. 41-57, 2010.

[2] A. Ziai and C. Menon, Comparison of regression models for estimation of isometric wrist joint torques using surface electromyography, in Journal of NeuroEngineering and Rehabilitation, vol. 8, pp. 56-67, 2011.

[3] O. Fukuda, T. Tsuji, M. Kaneko, and A. Otsuka, A Human-Assisting Manipulator Teleoperated by EMG Signals and Arm Motions, in IEEE Transactions on Robotics and Automation, vol. 19, no. 2, 2003.

[4] D. Nishikawa, W. Yu, H. Yokoi, and Y. Kakazu, EMG Prosthetic Hand Controller using Real-time Learning Method, in Proceedings of the IEEE International Conference on Systems, Man, and Cybernetics, (SMC), 1999.

[5] P. Shenoy, K. J. Miller, B. Crawford, and R. P. N. Rao, Online Electromyographic Control of a Robotic Prosthesis, in IEEE Transactions on Biomedical Engineering, vol. 55, no. 3, 2008.

[6] F. Sebelius, M. Axelsson, N. Danielsen, J. Schouenborg and T. Laurell, Real-time control of a virtual hand, in Technology and Disability, vol. 17, pp. 131-141, 2005.

[7] Y. Huang, K. B. Englehart, B. Hudgins, and A. D. C. Chan, A Gaussian Mixture Model Based Classification Scheme for Myoelectric Control of Powered Upper Limb Prostheses, in IEEE Transactions on Biomedical Engineering, vol. 52, no. 11, 2005.

[8] K. Kita, R. Kato, H. Yokoi, and T. Arai, Development ofAutonomous Assistive Devices-Analysis of change of human motion patterns, in IEEE International Symposium on Robot and Human Interactive Communication (RO-MAN), 2006

[9] C. Murray, Amputation, Prosthesis Use, and Phantom Limb Pain: An Interdisciplinary Perspective, in Springer Science+Business Media, chapter 2, 2010.

[10] J. Vogel, C. Castellini, and P. van der Smagt, EMG-Based Teleoperation and Manipulation with the DLR LWR-III, in IEEE/RSJ International Conference on Intelligent Robots and Systems, 2011.

[11] P.K. Artemiadis and K.J. Kyriakopoulos, A Switching Regime Model for the EMG-Based Control of a Robot Arm, in IEEE Transactions on Systems, Man, and Cybernetics Part B: Cybernetics, vol. 41, no. 1, 2011.

[12] N. Jiang, J. LG Vest-Nielsen, S. Muceli and D. Farina, EMG-based simultaneous and proportional estimation of wrist/hand kinematics in uni-lateral trans-radial amputees, in Journal of NeuroEngineering and Rehabilitation, vol. 9, 2012

[13] J.L Nielsen, S. Holmgaard, N. Jiang, K.B. Englehart, D. Farina, P.A. Parker, Simultaneous and proportional force estimation for multifunction myoelectric prostheses using mirrored bilateral training, in IEEE Transactions on Biomedical Engineering, vol. 8 no.3, 2011.

[14] R. C. Oldfield, The Assessment and Analysis of Handedness: the Edinburgh Inventory, Neuropsychologia, vol. 9, pp. 97-113, 1971.

[15] L. Masia, M. Casadio, G. Sandini and P. Morasso. Eye-Hand Coordination during Dynamic Visuomotor Rotations, in PloS one, vol. 9, no. 7004, 2009.

[16] L. Masia, V. Squeri, E. Burdet, G. Sandini and P. Morasso, Wrist Coordination in a Kinematically Redundant Stabilization Task, in IEEE Transaction on Haptics, vol. 5,no. 3, pp. 231239, 2012.

[17] C.C. Chang and C. J. Lin, LIBSVM: A library for support vector machines, ACM Transactions on Intelligent Systems and Technology, vol. 2:3, 2011 
TABLE II: Average and standard deviation of the $R^{2}$ index of the Abduction/Adduction angle estimation from the upper arm and forearm muscles for each of the 5 subjects. First row shows the $R^{2}$ index obtained for testing the algorithm on one position after training it on the same position. Rows 2 to 4 show the results of the generalization. The training in these cases was performed respectively on 1,2 or 3 arm positions and the generalization results were obtained by testing the algorithm on the remaining positions.

\begin{tabular}{|c|c|c|c|c|c|}
\hline \multicolumn{6}{|c|}{ Upper arm + Forearm Muscles: Abduction/Adduction Angle } \\
\hline Subjects & 1 & 2 & 3 & 4 & 5 \\
\hline Testing & $84.6 \pm 2.5$ & $74.9 \pm 4.2$ & $82.9 \pm 1.5$ & $80.3 \pm 3.2$ & $78.1 \pm 5.1$ \\
\hline Generalization (1 Vs all) & $67.9 \pm 6.9$ & $52.5 \pm 6.4$ & $54.9 \pm 8.6$ & $50.7 \pm 6.5$ & $63.4 \pm 2.6$ \\
\hline Generalization (2 Vs all) & $76.0 \pm 2.1$ & $54.5 \pm 5.2$ & $59.7 \pm 3.2$ & $57.3 \pm 6.9$ & $67.0 \pm 2.6$ \\
\hline Generalization (3 Vs all) & $79.3 \pm 2.0$ & $52.1 \pm 4.1$ & $62.0 \pm 4.0$ & $61.5 \pm 13.0$ & $69.4 \pm 1.0$ \\
\hline Generalization (4 Vs all) & $80.9 \pm 1.4$ & $57.2 \pm 4.0$ & $59.8 \pm 7.5$ & $68.2 \pm 6.2$ & $67.3 \pm 2.6$ \\
\hline Generalization (5 Vs all) & $81.5 \pm 1.5$ & $57.8 \pm 4.7$ & $64.3 \pm 4.4$ & $68.7 \pm 7.7$ & $70.7 \pm 3.4$ \\
\hline
\end{tabular}

TABLE III: Average and standard deviation of the $R^{2}$ index of the Flexion/Extension angle estimation from the upper arm and forearm muscles for each of the 5 subjects. First row shows the $R^{2}$ index obtained for testing the algorithm on one position after training it on the same position. Rows 2 to 4 show the results of the generalization. The training in these cases was performed respectively on 1,2 or 3 arm positions and the generalization results were obtained by testing the algorithm on the remaining positions.

\begin{tabular}{|c|c|c|c|c|c|}
\hline \multicolumn{7}{|c|}{ Upper arm + Forearm Muscles: Flexion/Extension Angle } \\
\hline Subjects & 1 & 2 & 3 & 4 & 5 \\
\hline Testing & $86.9 \pm 2.2$ & $77.6 \pm 4.6$ & $78.8 \pm 4.2$ & $58.1 \pm 10.2$ & $74.4 \pm 5.6$ \\
\hline Generalization (1 Vs all) & $70.1 \pm 3.2$ & $56.7 \pm 7.0$ & $40.2 \pm 7.7$ & $28.7 \pm 10.4$ & $49.5 \pm 3.7$ \\
\hline Generalization (2 Vs all) & $68.4 \pm 4.7$ & $55.6 \pm 5.2$ & $45.8 \pm 9.0$ & $31.2 \pm 12.9$ & $53.9 \pm 3.7$ \\
\hline Generalization (3 Vs all) & $69.6 \pm 6.0$ & $62.7 \pm 3.3$ & $54.3 \pm 6.5$ & $37.4 \pm 6.2$ & $52.1 \pm 4.2$ \\
\hline Generalization (4 Vs all) & $71.6 \pm 3.4$ & $60.8 \pm 4.9$ & $53.5 \pm 10.6$ & $37.0 \pm 7.8$ & $56.2 \pm 4.0$ \\
\hline Generalization (5 Vs all) & $71.0 \pm 5.6$ & $64.0 \pm 6.0$ & $57.7 \pm 5.6$ & $43.4 \pm 7.5$ & $57.2 \pm 5.6$ \\
\hline
\end{tabular}

TABLE IV: Average and standard deviation of the $R^{2}$ index of the Abduction/Adduction and Flexion/Extension angles' estimation from the upper arm and forearm muscles across the 5 subjects.

\begin{tabular}{|c|c|c|c|c|c|c|}
\hline & \multicolumn{3}{|c|}{ Abduction/Adduction } & \multicolumn{3}{|c|}{ Flexion/Extension } \\
\hline & Upper arm + Forearm & Upper arm & Forearm & Upper arm + Forearm & Upper arm & Forearm \\
\hline Testing & $80.2 \pm 3.8$ & $73.3 \pm 3.5$ & $75.0 \pm 4.1$ & $75.2 \pm 10.6$ & $62.5 \pm 8.2$ & $69.9 \pm 13.4$ \\
\hline Generalization (1 Vs all) & $57.9 \pm 7.4$ & $46.3 \pm 14.8$ & $52.7 \pm 2.6$ & $49.1 \pm 15.8$ & $31.5 \pm 6.6$ & $41.9 \pm 21.7$ \\
\hline Generalization (2 Vs all) & $62.9 \pm 8.7$ & $51.2 \pm 11.9$ & $57.8 \pm 3.2$ & $51.0 \pm 13.7$ & $33.8 \pm 6.8$ & $47.4 \pm 17.7$ \\
\hline Generalization (3 Vs all) & $64.9 \pm 10.2$ & $54.2 \pm 8.4$ & $62.3 \pm 4.0$ & $55.2 \pm 12.2$ & $37.4 \pm 7.4$ & $49.0 \pm 19.4$ \\
\hline Generalization (4 Vs all) & $66.7 \pm 9.2$ & $56.9 \pm 7.2$ & $64.2 \pm 3.9$ & $55.8 \pm 12.6$ & $40.4 \pm 4.8$ & $50.5 \pm 16.6$ \\
\hline Generalization (5 Vs all) & $68.6 \pm 8.7$ & $57.0 \pm 9.9$ & $64.5 \pm 2.5$ & $58.6 \pm 10.2$ & $41.4 \pm 5.7$ & $51.7 \pm 18.7$ \\
\hline
\end{tabular}

TABLE V: $R^{2}$ index of the Abduction/Adduction and Flexion/Extension angles' estimation from the upper arm and forearm muscles when testing on data collected from day 1 .

\begin{tabular}{|c|c|c|c|c|c|c|}
\hline & \multicolumn{6}{|c|}{ Testing on day 1 data } \\
\hline Wrist joint angles & \multicolumn{3}{|c|}{ Abduction/Adduction } & \multicolumn{3}{|c|}{ Flexion/Extension } \\
\hline Muscles & Upper arm + Forearm & Upper arm & Forearm & Upper arm + Forearm & Upper arm & Forearm \\
\hline Trained on day 2 data & 1.3 & 35.8 & 2.6 & 12.5 & 33.5 & 46.0 \\
\hline Trained on day 3 data & 44.4 & 51.2 & 30.3 & 51.8 & 15.7 & 51.6 \\
\hline Trained on days 2 and 3 data & 56.7 & 59.3 & 14.8 & 58.1 & 20.8 & 41.8 \\
\hline
\end{tabular}

TABLE VI: $R^{2}$ index of the Abduction/Adduction and Flexion/Extension angles' estimation from the upper arm and forearm muscles when testing on data collected from day 2.

\begin{tabular}{|c|c|c|c|c|c|c|}
\hline & \multicolumn{6}{|c|}{ Testing on day 2 data } \\
\hline Wrist joint angles & \multicolumn{3}{|c|}{ Abduction/Adduction } & \multicolumn{3}{|c|}{ Flexion/Extension } \\
\hline Muscles & Upper arm + Forearm & Upper arm & Forearm & Upper arm + Forearm & Upper arm & Forearm \\
\hline Trained on day 1 data & 55.2 & 57.0 & 38.0 & 4.4 & 34.9 & 8.3 \\
\hline Trained on day 3 data & 42.3 & 36.0 & 22.2 & 0.2 & 20.2 & 0.6 \\
\hline Training on days 1 and 3 data & 52.1 & 48.8 & 30.7 & 0.12 & 32.6 & 1.2 \\
\hline
\end{tabular}

TABLE VII: $R^{2}$ index of the Abduction/Adduction and Flexion/Extension angles' estimation from the upper arm and forearm muscles when testing on data collected from day 3 .

\begin{tabular}{|c|c|c|c|c|c|c|}
\hline & \multicolumn{4}{|c|}{ Testing on day 3 data } \\
\hline Wrist joint angles & \multicolumn{2}{|c|}{ Abduction/Adduction } & \multicolumn{4}{c|}{ Flexion/Extension } \\
\hline Muscles & Upper arm + Forearm & Upper arm & Forearm & Upper arm + Forearm & Upper arm & Forearm \\
\hline Trained on day 1 data & 62.7 & 53.5 & 43.8 & 43.2 & 25.8 & 46.4 \\
\hline Trained on day 2 data & 11.1 & 34.9 & 6.1 & 18.7 & 30.0 & 2.3 \\
\hline Trained on days 1 and 2 & 55.3 & 53.5 & 37.7 & 41.7 & 23.5 & 31.9 \\
\hline
\end{tabular}

